(C)2002 The American Physical Society. All rights reserved. Access to this work was provided by the University of Maryland, Baltimore County (UMBC) ScholarWorks@UMBC digital repository on the Maryland Shared Open Access (MD-SOAR) platform.

Please provide feedback

Please support the ScholarWorks@UMBC repository by emailing scholarworks-group@umbc.edu and

telling us

what having access to this work means to you and why it's important to you. Thank you. 


\title{
Demonstration of Nondeterministic Quantum Logic Operations Using Linear Optical Elements
}

\author{
T. B. Pittman, B. C. Jacobs, and J. D. Franson \\ The Johns Hopkins University, Applied Physics Laboratory, Laurel, Maryland 20723
}

(Received 2 November 2001; published 6 June 2002)

\begin{abstract}
Knill, Laflamme, and Milburn [Nature (London) 409, 46 (2001)] recently showed that nondeterministic quantum logic operations could be performed using linear optical elements, additional photons (ancilla), and postselection based on the output of single-photon detectors. Here we report the experimental demonstration of two logic devices of this kind, a destructive controlled-NOT (CNOT) gate and a quantum parity check. These two devices can be combined with a pair of entangled photons to implement a conventional (nondestructive) CNOT that succeeds with a probability of $\frac{1}{4}$.
\end{abstract}

DOI: $10.1103 /$ PhysRevLett.88.257902

PACS numbers: 03.67.Lx, 42.25.Hz, 42.50.Ct

One of the main difficulties in any optical approach to quantum information processing is that nonlinear interactions between single photons are required to implement logic devices that operate with $100 \%$ efficiency. Recently, however, Knill, Laflamme, and Milburn (KLM) [1] showed that probabilistic quantum logic operations could be performed using only linear optical elements, additional photons (ancilla), and postselection based on the output of single-photon detectors. These devices succeed in producing the desired logical output with a probability that can approach unity, and they may form the basis for a scalable approach to quantum computing.

Here we report the experimental demonstration of two logic devices of this kind, a destructive controlled-NOT (CNOT) gate and a quantum parity check [2]. The output of the destructive CNOT (DCNOT) gate is identical to that of a conventional (nondestructive) CNOT gate except that the information associated with the control photon is destroyed during the operation. However, the destructive CNOT gate and quantum parity check demonstrated here can be combined with a pair of entangled photons to implement a conventional CNOT gate that succeeds with a probability of $\frac{1}{4}$ [2].

In the original approach suggested by KLM [1], the value of each qubit is represented by a single photon that may be located in one of two paths, such as two optical fibers. One path represents a logical value of 0 while the other path represents a value of 1 . The logic operations involve interference between different optical paths, which can be very sensitive to thermally induced phase shifts. In contrast, we use polarization-encoded qubits [3] in which the values of the qubits are represented by the two linear states of polarization of a single photon, with a horizontal polarization state $|H\rangle$ representing the value 0 and a vertical polarization state $|V\rangle$ representing the value 1 . The use of polarization-encoded qubits can eliminate the need for any interference between two different optical paths [4], which may be an advantage in practical applications.

In an earlier theory paper [2], we showed that polarization-encoded qubits and postselection could be used to implement a number of nondeterministic quantum logic operations, including the quantum parity check and destructive CNOT gate shown in Fig. 1. In each of these devices, two input qubits in the form of two single photons are incident on a polarizing beam splitter that completely transmits horizontally polarized photons and totally reflects vertically polarized photons. A polarizationsensitive detector in output path $2^{\prime}$ detects any photons in either the horizontal-vertical (HV) computational basis or another basis rotated by $45^{\circ}$. As shown in the insets, the polarization-sensitive detectors can be implemented using another polarizing beam splitter and two single-photon detectors. The output of the devices is accepted only for those cases in which the detector registers one and only one photon. This postselection process provides the origin of the nonlinearity required for the logic operations, since the detection process is inherently nonlinear.

Although the physical structure of these two devices is similar, the operation and desired output of each is very different. The operation of the quantum parity check is the

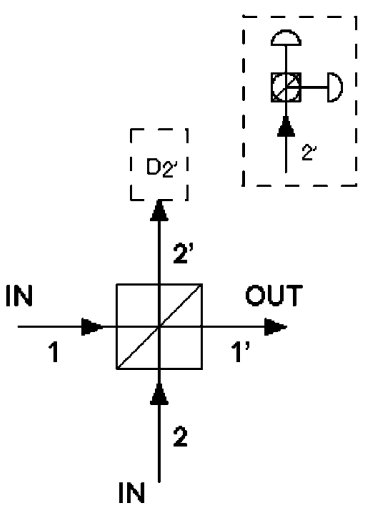

(a) quantum parity check

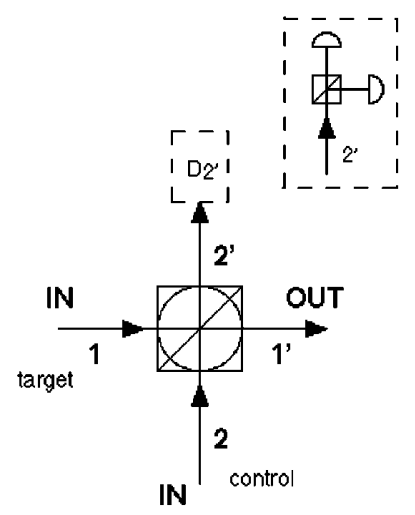

(b) destructive-CNOT gate
FIG. 1. Implementation of a quantum parity check (a) and a destructive CNOT gate (b) using polarizing beam splitters. The polarizing beam splitter (PBS) is oriented in the HV computational basis for the parity check and rotated by $45^{\circ}$ for the destructive CNOT. The polarization-sensitive detector $D_{2^{\prime}}$ consists of a second polarizing beam splitter and two single-photon detectors. It is oriented in the HV basis for the destructive CNOT and rotated by $45^{\circ}$ for the parity check. 
most straightforward and will be described first, although the demonstration of the destructive CNOT gate is the most important result presented here. The goal of the quantum parity check shown in Fig. 1a is to transfer the value of the input qubit in path 1 to the output in path $1^{\prime}$ provided that its value is the same as that of the second input qubit in path 2. If the qubits have different values, the device fails and produces no output. The parity-check operation can be understood from the basic properties of a polarizing beam splitter (PBS) [5]. If only one photon is to be detected, both of the incident photons must be transmitted or both must be reflected. In either case, the polarizations and the corresponding values of the qubits must be the same. This ability to compare the polarizations of two photons has been proposed for use in entanglement purification [5] and a variety of other quantum information processing applications [6-9].

The parity-check operation described above is essentially classical in nature, but quantum-computing applications require that it be performed without measuring or determining the values of either qubit. For the probabilistic quantum parity check illustrated in Fig. 1a, this is accomplished by orienting the polarization-sensitive detector in a basis rotated by $45^{\circ}$, which essentially erases any information regarding the value of either of the input qubits. When the inputs consist of an arbitrary superposition of states, this quantum erasure $[10,11]$ technique combined with the postselection process maintains the required coherence of the probability amplitudes, as will be described below.

The implementation of the destructive CNOT gate shown in Fig. $1 \mathrm{~b}$ is similar to the quantum parity check, except that the polarizing beam splitter is oriented in a basis rotated by $45^{\circ}$ and the polarization-sensitive detector is oriented in the HV basis. The goal of the destructive CNOT gate is to flip the logical value of the target qubit (e.g., $0 \leftrightarrow 1$ ) if the control qubit has the value 1 and to do nothing if the control qubit has the value 0 . The expected output of the gate shown in Fig. 1b is that of a conventional CNOT, except that the information contained in the control qubit is destroyed by the polarization-sensitive detector. The operation of the destructive CNOT gate can be understood by expanding the input qubit polarization states in the $45^{\circ}$ basis of the polarizing beam splitter and then reexpressing the output states in the HV basis of the detector. It can be shown [2] that an arbitrary target state will be flipped if the control photon in mode 2 was vertically polarized (logical value 1) but it will be unaltered for a horizontally polarized control photon (logical value 0):

$$
\begin{array}{ll}
\text { Control }=1: & \alpha|H\rangle_{1}+\beta|V\rangle_{1} \rightarrow \alpha|V\rangle_{1^{\prime}}+\beta|H\rangle_{1^{\prime}}, \\
\text { Control }=0: & \alpha|H\rangle_{1}+\beta|V\rangle_{1} \rightarrow \alpha|H\rangle_{1^{\prime}}+\beta|V\rangle_{1^{\prime}} .
\end{array}
$$

Because of the similarity of these two devices, we were able to demonstrate both logic operations using a single ex-

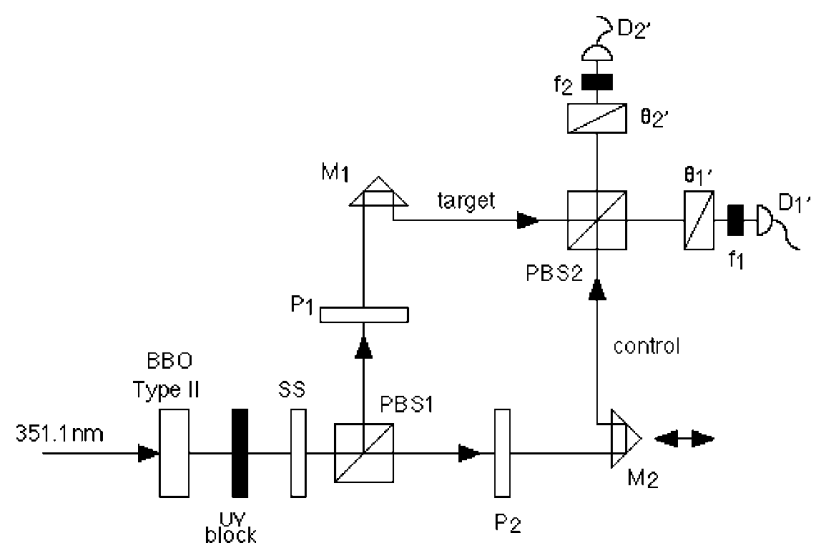

FIG. 2. A simplified outline of the experimental apparatus used to demonstrate the quantum parity check and destructive CNOT operations of Fig. 1. A $1.0 \mathrm{~mm}$ thick BBO down-conversion crystal (type-II) was pumped by roughly $30 \mathrm{~mW}$ of the $351.1 \mathrm{~nm}$ line of an argon-ion laser. PBS1 and PBS2 were polarizing beam splitters, while $M_{1}$ and $M_{2}$ were mirrors mounted on translation stages. Half-wave plates $P_{1}$ and $P_{2}$ were located in each path, while $\theta_{1^{\prime}}$ and $\theta_{2^{\prime}}$ were polarization analyzers in front of detectors $D_{1^{\prime}}$ and $D_{2^{\prime}} . f_{1}$ and $f_{2}$ were $10 \mathrm{~nm}$ FWHM bandpass filters centered at $702 \mathrm{~nm}$, and SS was a birefringent element for ShihSergienko compensation [12].

perimental setup, which is shown schematically in Fig. 2. Here the polarizing beam splitters of Figs. 1a and 1b correspond to the second polarizing beam splitter, PBS2, in Fig. 2. The initial polarizing beam splitter, PBS1, was simply used to separate the two photons produced in a type-II parametric down-conversion $[12,13]$ crystal (BBO), which produces pairs of photons, one polarized vertically, and the other polarized horizontally. The two photons were then directed along two different paths towards PBS2. The two paths were adjusted to the same length using mirrors on translation stages, which was necessary in order to ensure that both photons arrived at the second beam splitter at the same time [14]. The polarization of either photon could be rotated into any desired orientation using half-wave birefringent plates located in each path.

The polarization-sensitive detectors of Figs. 1a and $1 \mathrm{~b}$ were implemented using a rotatable polarization analyzer $\theta_{2^{\prime}}$ and one single-photon detector in output path $2^{\prime}$. In contrast to the polarization-sensitive detectors of Fig. 1, this arrangement could detect only one of the possible output polarization states and reduced the probability of success from $\frac{1}{2}$ to $\frac{1}{4}$ [2]. Another polarization analyzer $\theta_{1^{\prime}}$ and a second single-photon detector were used to measure the polarization state of the photons in the logical output path $1^{\prime}$, which allowed a comparison between the expected and actual outputs of the devices. Since only two photons were incident at any given time, the postselection process described above was equivalent to monitoring the rate of coincident detection events as a function of various combinations of wave plate and analyzer orientations.

The experimental results from the quantum parity-check operation are summarized in Figs. 3 and 4. The logical 

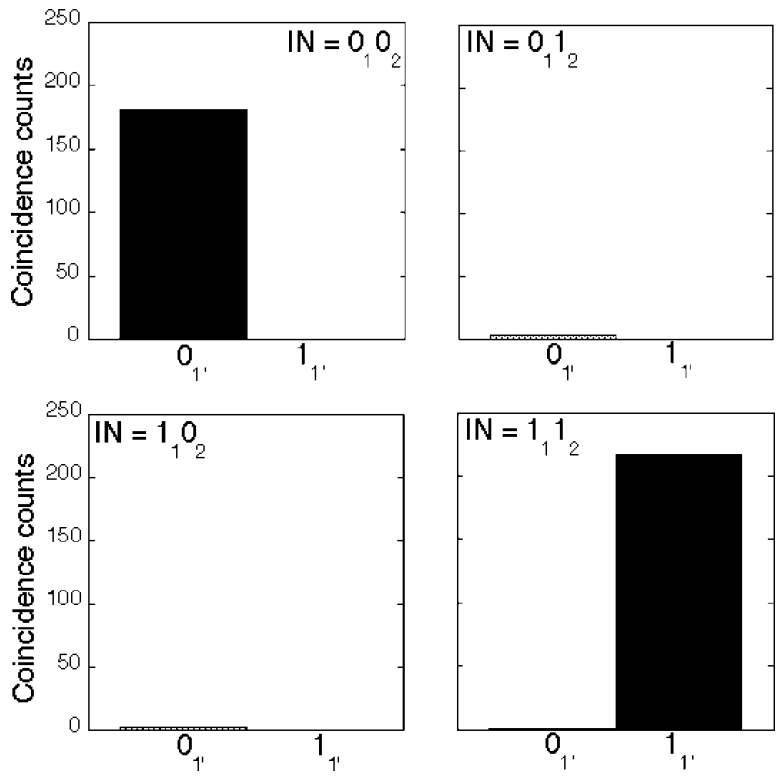

FIG. 3. Logical truth table summarizing the results from the quantum parity check. The number of coincidence events per minute corresponding to 0 or 1 that were obtained for all possible values of the input qubits is shown. The results are consistent with what would be expected from a parity-check operation.

truth table of Fig. 3 shows the relative probability of obtaining an output value of 0 or 1 for all of the possible combinations of input values. The half-wave plates were oriented in this case to give input photons with horizontal or vertical polarizations ( 0 or 1$)$, and the polarization analyzer $\theta_{1^{\prime}}$ was oriented horizontally or vertically. It can be seen that the results obtained agree with what would be expected from a parity check to within an error on the order of $1 \%$.

A more intriguing property of the quantum parity check is illustrated in Fig. 4, where the wave plates were adjusted so that input 2 was in the state $\mid$ in $\rangle_{2}=\frac{1}{\sqrt{2}}(|0\rangle+|1\rangle)$. As we showed in Ref. [2], an arbitrary input state in path 1 is expected to be coherently transferred into output path $1^{\prime}$ for this choice of input 2. It is interesting to note that the polarizing beam splitter PBS2 transmits the horizontal component of input 1 into output path $1^{\prime}$ as required, but it totally reflects the vertical component into detector $D_{2^{\prime}}$, where it is destroyed. As a result, the device must somehow replace the arbitrary vertical component from input 1 with a vertical component from input 2 (which is reflected into output path $1^{\prime}$ ) even though that component initially had a fixed value of $\frac{1}{\sqrt{2}}$. In essence, the postselection process makes the polarizing beam splitter transparent to all components of input 1 . For the data shown in Fig. 4, input 1 was arbitrarily chosen to be in the state $\mid$ in $\rangle_{1}=0.94|0\rangle+0.34|1\rangle$, which corresponds to a photon polarized at an angle of approximately $20^{\circ}$. It can be seen that the output state corresponds to the same polarization to within the experimental error, which demonstrates the coherent nature of the quantum parity-check operation.

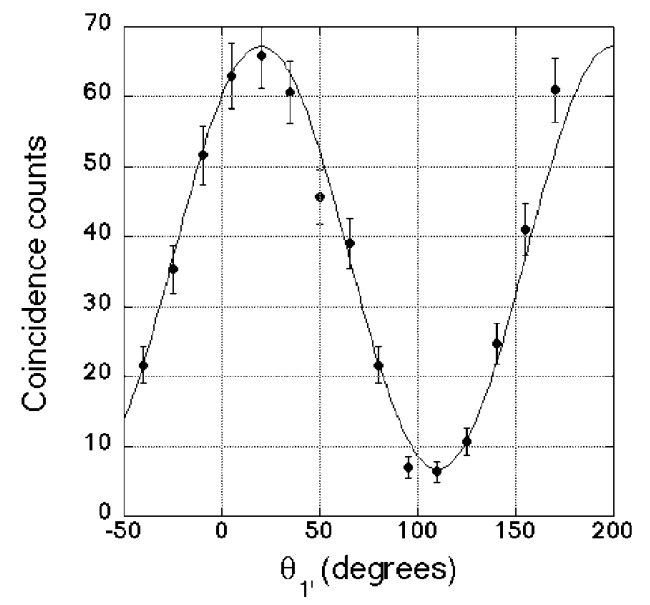

FIG. 4. Experimental test of the coherence of the output of the quantum parity check. The number of coincidence events per minute is plotted as a function of the setting of polarization analyzer $\theta_{1^{\prime}}$ for an input 1 state corresponding to a polarization of $20^{\circ}$. The solid curve represents an empirical fit to the data. The measurements are consistent with an output state with the same polarization, which demonstrates the coherence of the parity check when acting on superposition states.

The main result of this Letter is shown in Fig. 5, which shows a logical truth table summarizing the experimental results for the destructive CNOT gate. Rather than physically rotating the polarizing beam splitter through a $45^{\circ}$ angle as shown in Fig. 1b, it was more convenient to rotate the incident photons and the detector bases using the half-wave plates. As mentioned above, the intended function of the destructive CNOT gate is to flip the target bit if and only if the control bit is equal to 1 . It can be seen from Fig. 5 that our destructive CNOT gate performs this operation with an average error of approximately $17 \%$ for the case where all of the input qubits have the value 0 or 1. The mean error for the current setup when averaged over all possible input states is approximately $8 \%$. These errors were largely due to the optical quality of the polarizing beam splitters, which were of commercial grade and had distortions on the order of $\frac{1}{4}$ wave. Much lower error rates could be obtained using custom-made polarizing beam splitters and single mode optical fibers. In any event, the controlled target state-flipping operation of the destructive CNOT is clearly evident from the data shown in Fig. 5.

It can be seen that the errors in the destructive CNOT data of Fig. 5 are much larger than the errors in the quantum parity-check data of Fig. 3, which can be understood as follows. When the polarizations of the photons input to the quantum parity check are either horizontal or vertical (and not a superposition state), the output does not depend on quantum interference effects and the results are unaffected by misalignments or birefringence. The destructive CNOT data of Fig. 5, however, does depend on higher-order interference between the photons and is thus sensitive to misalignments and birefringence. The situation is reversed 

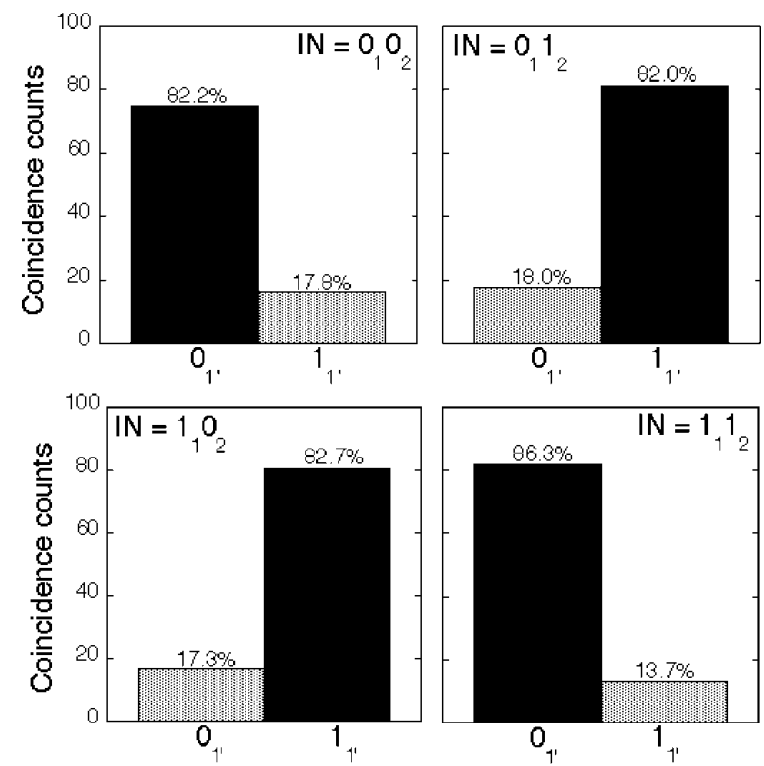

FIG. 5. Logical truth table summarizing the results obtained from the destructive CNOT. The number of coincidence events per minute corresponding to 0 or 1 that were obtained for all possible values of the input qubits is shown. The results show that the destructive CNOT flips the value of the target qubit when the control qubit has a value of 1 , as desired. The errors shown here correspond to the worst case and could be greatly reduced using custom-made optics or optical fibers.

for superposition states, in which case the errors in the parity check can be larger than those in the destructive CNOT, and the average errors are the same for the two devices.

The destructive CNOT operation was also tested using superpositions of input states. Coherent results similar to those of Fig. 4 were obtained but are not included here. Once again, this shows that the operation was performed without measuring the values of the qubits [2].

The results presented here demonstrate that certain nondeterministic quantum logic operations can be performed in a straightforward manner using linear optical elements [1]. As we have shown earlier [2], the quantum parity check and destructive CNOT described above can be combined with a pair of entangled photons to implement a nondestructive CNOT operation with a probability of success of $\frac{1}{4}$. Experiments of that kind will require the production of four photons in the same spatial and temporal mode [15-17], whereas our current experiments required only two photons. Zeilinger's group has recently demonstrated [18] a method for producing the necessary four-photon state, so that the demonstration of a full nondeterministic CNOT gate should be feasible. A more difficult challenge for practical applications is the requirement that the proba- bility of success be on the order of unity as is required by the current state of quantum error correction [19]. In principle, KLM [1] have shown that it is possible to use additional ancilla and detectors to achieve a success rate arbitrarily close to one. We expect that further work in that area will be required in order to develop a practical approach to quantum computing. In any event, the experimental results presented here may provide a first step towards that goal.

This work was supported by the U.S. Office of Naval Research and by Independent Research and Development funds. We acknowledge valuable discussions with M. Donegan and M. Fitch.

[1] E. Knill, R. Laflamme, and G. J. Milburn, Nature (London) 409, 46 (2001).

[2] T. B. Pittman, B. C. Jacobs, and J. D. Franson, Phys. Rev. A 64, 062311 (2001).

[3] D. Bouwmeester, A. Ekert, and A. Zeilinger, The Physics of Quantum Information (Springer-Verlag, Berlin, 2000).

[4] M. Koashi, T. Yamamoto, and N. Imoto, Phys. Rev. A 63, 030301 (2001).

[5] J. W. Pan, C. Simon, C. Brukner, and A. Zeilinger, Nature (London) 410, 1067 (2001).

[6] J. W. Pan and A. Zeilinger, Phys. Rev. A 57, 2208 (1998).

[7] D. Bouwmeester, J. W. Pan, M. Daniell, H. Weinfurter, and A. Zeilinger, Phys. Rev. Lett. 82, 1345 (1999).

[8] D. Bouwmeester, Phys. Rev. A 63, 040301 (2001).

[9] T. Yamamoto, M. Koashi, and N. Imoto, Phys. Rev. A 64, 012304 (2001).

[10] M. O. Scully and K. Druhl, Phys. Rev. A 25, 2208 (1982).

[11] P. G. Kwiat, A. M. Steinberg, and R. Y. Chiao, Phys. Rev. A 45, 7729 (1992).

[12] Y. H. Shih and A. V. Sergienko, Phys. Lett. A 186, 29 (1994).

[13] M. H. Rubin, D. N. Klyshko, Y.H. Shih, and A. V. Sergienko, Phys. Rev. A 50, 5122 (1994).

[14] T. B. Pittman, D. V. Strekalov, A. Migdall, M. H. Rubin, A. V. Sergienko, and Y.H. Shih, Phys. Rev. Lett. 77, 1917 (1996).

[15] M. Zukowski, A. Zeilinger, and H. Weinfurter, Ann. N.Y. Acad. Sci. 755, 91 (1995).

[16] A. Zeilinger, M. A. Horne, H. Weinfurter, and M. Zukowski, Phys. Rev. Lett. 78, 3031 (1997).

[17] Z. Y. Ou, Quantum Semiclass. Opt. 9, 599 (1997).

[18] J. W. Pan, M. Daniell, S. Gasparoni, G. Weihs, and A. Zelinger, Phys. Rev. Lett. 86, 4435 (2001).

[19] M. A. Nielsen and I. L. Chuang, Quantum Computing and Quantum Information (Cambridge University Press, Cambridge, U.K., 2000). 